\title{
EL CONCEPTO DE "HÁBITAT DIGNO" COMO META DE UNA POLÍTICA INTEGRAL DE ÁREAS URBANAS DEFICITARIAS CRÍTICAS, PARA LA INTEGRACIÓN SOCIAL DESDE LOS DERECHOS HUMANOS ${ }^{1}$
}

Miguel Ángel Barreto²

\section{Introducción}

Actuar desde el reconocimiento y el respeto de los derechos humanos implica para el Estado, en primer lugar, reconocer umbrales mínimos de satisfacción en un conjunto de necesidades humanas básicas para toda la sociedad y, en segundo lugar, asumir el compromiso de empezar a garantizarlos a todos aquellos hogares que por diferentes razones se encuentran excluidos de la satisfacción de estas necesidades, como vía de integración social.

1 Este trabajo es resultado de investigaciones realizadas como Director de los proyectos: PIP 112-200801-00634 (CONICET), PICT0-UNNE 2007-00100 (ANPCyT) y PI 041-07 (SGCyT-UNNE). Agradezco profundamente a los siguientes integrantes de estos proyectos por el enriquecimiento intelectual mutuo dado en el trabajo en equipo: Laura ALCALÁ, Marta GIRÓ, Andrea BENÍTEZ, María E. FERNÁNDEZ, María B. PELLI, Venettia ROMAGNOLI y especialmente al Arq. Víctor PELLI.
Cabe precisar que se entiende aquí a los derechos humanos como categoría integradora de los diferentes derechos (civiles, políticos, sociales, económicos, culturales, ambientales, etc.), conquistados y consagrados en distintas legislaciones y pactos internacionales. Según el Punto 2 del Artículo I de la Carta Mundial por el Derecho a la Ciudad, los derechos humanos incluyen: "los derechos al trabajo en condiciones equitativas y satisfactorias; a fundar y afi-

2 Argentino, Arquitecto, Magíster y Doctor en Antropología Social. Investigador Adjunto del CONICET y la SGCyT-UNNE (Argentina). Profesor Adjunto de la Facultad de Arquitectura y Urbanismo de la Universidad Nacional del Nordeste (FAUUNNE). Instituto de Investigación y Desarrollo en Vivienda (IIDVI, FAU, UNNE).

Correo electrónico: mabarreto@arnet.com.ar. 
liarse a sindicatos; a seguridad social, salud pública, agua potable, energía eléctrica, transporte público y otros servicios sociales; a alimentación, vestido y vivienda adecuada; a educación pública de calidad y la cultura; a la información, la participación política, la convivencia pacífica y el acceso a la justicia; a organizarse, reunirse y manifestarse. Incluye también el respeto a las minorías y la pluralidad étnica, racial, sexual y cultural y el respeto a los migrantes"3.

Según esta Carta, "El Derecho a la Ciudad es interdependiente de todos los derechos humanos internacionalmente reconocidos, concebidos integralmente, e incluye, por tanto, todos los derechos civiles, políticos, económicos, sociales, culturales y ambientales que ya están reglamentados en los tratados internacionales de derechos humanos." ${ }^{4}$ Por otra parte, se entiende aquí por integración social a una situación en la que los miembros de los hogares de una sociedad (cualquiera sea su condición de edad, género o étnica) estén insertos dentro del sistema formal (en condición activa o pasiva) y cuenten con protecciones sociales básicas para acceder a los servicios sociales esenciales, de salud, educación, vivienda, seguridad, ocio y recreación, etc., de manera adecuada en relación con las condiciones de vida generales de la sociedad de pertenencias.
Con relación a las necesidades habitacionales, el Estado argentino desde hace tiempo reconoce algunos estándares de calidad de materiales, de construcción e instalaciones dentro de la vivienda, así como de ciertos servicios y grados de hacinamientos, que definen un umbral entre las condiciones habitacionales adecuadas y deficitarias de la población. Este umbral determina las condiciones de una vivienda digna $O$ adecuada (consagrada como derecho en la Constitución Argentina) y traza una línea que demarca los hogares que necesitan mejorar dichas condiciones, y por ende, determina el déficit habitacional.

Entendiendo que el problema de quienes viven en áreas urbanas deficitarias críticas -configuradas en torno a lo que en Argentina se denominan comúnmente "villas" y "asentamientos"-, excede ampliamente la carencia de una vivienda adecuada y considerando que con mucha frecuencia, desde el Estado se interviene desde la política habitacional sobre este problema con un enfoque muy sectorial, el presente trabajo propone y analiza el concepto de Hábitat Digno como meta de diseño de una política más integral para el problema.

Con esta finalidad, el trabajo analiza, primero, algunas cuestiones estructurales relacionadas con el incremento del problema en Argentina; en segun-

3 Carta mundial por el derecho a la ciudad, 2005: 2.

$40 p$ cit. Estas ideas en torno a las citas de esta Carta fueron citadas originalmente en BARRETO, $2008^{\mathrm{a}}$.

5 Barreto, 2005

162 revista invi № 69 / Agosto 2010 / Volumen № 25: 161-187 
do lugar, los enfoques predominantes de las políticas en las últimas décadas; en tercer lugar, realiza una caracterización del problema para entender su complejidad a partir del conjunto de factores interrelacionados que intervienen en el mismo; y finalmente, desarrolla el concepto de Hábitat Digno como meta de una política integral, consistente con la perspectiva de los derechos humanos aludida.

\section{Urbanización, reforma del Estado, pobreza e incremento de las áreas urbanas deficitarias críticas en las últimas décadas en Argentina}

La población de la mayoría de las ciudades argentinas ha experimentado en las últimas cuatro décadas un importante crecimiento, debido, en parte, al incremento vegetativo, pero principalmente, al sostenido proceso de urbanización y de concentración urbana ocurrido. ${ }^{6}$ A la vez, este proceso de

6 Según el INDEC, en 1970 el 79 \% de la población Argentina vivía en centros urbanos de más de 2.000 habitantes, en 1980 este valor ascendió al $83 \%$, en 1991 al 87,2 \% y en el año 2001 al 89,4 $\%$. Este último porcentaje representaba a 32.431 .950 habitantes sobre un total de 36.260 .130 . concentración urbana afectó más a las ciudades intermedias ${ }^{7}$ que a las grandes ciudades y al aglomerado Gran Buenos Aires; por este motivo comenzó a revertirse parcialmente el histórico desequilibrio de la población urbana argentina. ${ }^{8}$ Sin embargo, estos indicadores no sólo muestran que Argentina es en la actualidad uno de los países con mayor porcentaje de población urbana del mundo, sino también que continúa siendo muy desequilibrado en cuanto a la distribución territorial de su población, ya que si bien cuenta con 92 ciudades de más de 32.000 habitantes, concentra en sus cuatro principales aglomerados urbanos al 45,75\% de la población urbana total del país, disponiendo de extensas áreas rurales de su vasto territorio, apenas habitadas y en continuo despoblamiento (Figura 1).

Por otra parte, durante este periodo, las políticas de liberalización, iniciadas en la última dictadura militar (1976-1982) y profundizadas durante los años $90^{9}$ en el marco de la Reforma del Estado impulsada por el "Consenso de Washington", produ-

7 Basualdo, 2001.

8 De acuerdo con esta misma fuente, el porcentaje de población que vivía en ciudades de más de 100.000 habitantes era en 1980 de $57,4 \%$, en 1991 de $60,6 \%$ y en 2001 de $62,5 \%$.

9 En 1980 el porcentaje de población que habitaba en los cuatro aglomerados más importantes, Gran Buenos Aires, Gran Córdoba, Gran Rosario y Gran Mendoza, era de 44,9\% y descendió a $44,1 \%$ en 1991 y a 42,5 en 2001, disminuyendo, por lo tanto, el índice de primacía urbana de 3,9 en 1980 a 3,6 en 2001 (INDEC). 


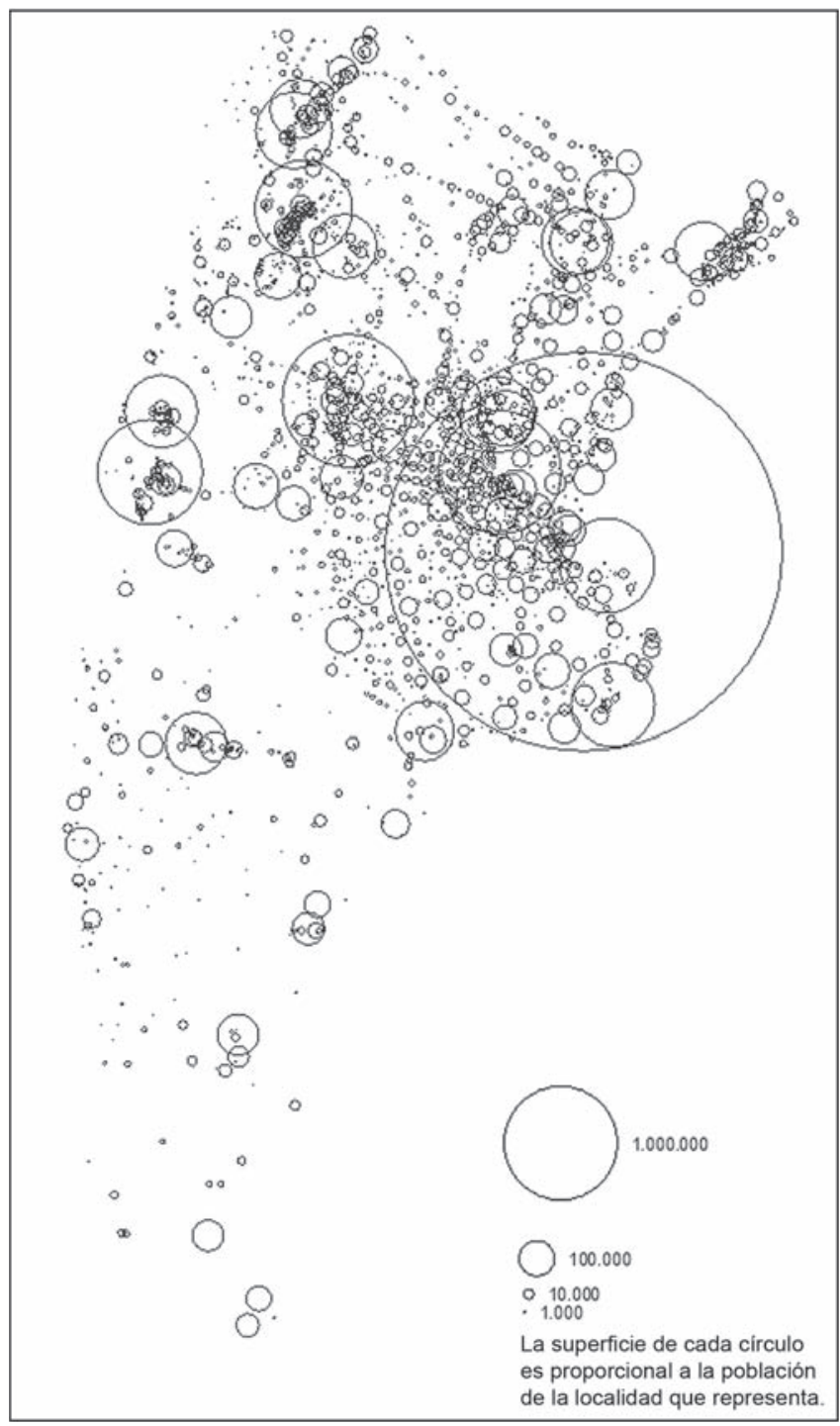

POBLACIÓN DE ARGENTINA EN LOCALIDADES DE MÁS DE 1.000 HABITANTES
FIGURA 1

Fuente: Ministerio del Interior. Elaborado con datos de INDEC: Censo Nacional de Población Hogares y Vivienda 2001. 
jeron un incremento sostenido de la desigualdad en la distribución del ingreso, ${ }^{10}$ la que, en términos concretos, se manifestó a partir de fines de los años 80 en una permanencia variable pero constante (estructural) de altos valores de pobreza y desocupación (Tabla 1).

\section{EVOLUCIÓN DE LA INDIGENCIA, LA POBREZA Y LA DESOCUPACIÓN EN EL GBA $(1988 \text { - 2002) })^{11}$}

\begin{tabular}{|c|c|c|c|}
\hline \multirow{2}{*}{$\begin{array}{l}\text { Fecha de } \\
\text { relevamiento }\end{array}$} & \multicolumn{2}{|c|}{ Personas } & \multirow{2}{*}{$\begin{array}{l}\text { Tasa de } \\
\text { desocupación }\end{array}$} \\
\hline & $\begin{array}{l}\text { bajo la línea } \\
\text { de indigencia }\end{array}$ & $\begin{array}{l}\text { bajo la línea } \\
\text { de pobreza }\end{array}$ & \\
\hline may-88 & 8,6 & 29,8 & 6,3 \\
\hline may-90 & 12,5 & 42,5 & 8,6 \\
\hline may-93 & 3,6 & 17,7 & 10,6 \\
\hline may-95 & 5,7 & 22,2 & 20,2 \\
\hline may-99 & 7,6 & 27,1 & 15,6 \\
\hline oct-02 & 24,7 & 54,3 & 18,8 \\
\hline
\end{tabular}

TABLA 1

Fuente: INDEC, sobre la base de la Encuesta Permanente de Hogares.

10 Según ALTAMIR y BECCARIA (2000: 432), el coeficiente de Gini en el área metropolitana del Gran Buenos Aires (única fuente disponible histórica) en 1974 era de 0,361 ; en 1980 era de 0,416 ; en 1986 era de 0,419; en 1986 era de 0,419; en 1990 era de 0,437 y en 1997 era de 0,446.

11 Se hace referencia solamente al Gran Buenos Aires, porque recién a partir del año 2001 la Encuesta Permanente de Hogares del INDEC se hizo extensiva a los 28 aglomerados más importantes del país.
Esta combinación de procesos de concentración urbana, de incremento estructural de la pobreza y de la desocupación, y de políticas que implicaron eliminación de derechos sociales, mercantilización de servicios públicos básicos y desinversión estructural en los sectores de menores recursos, tuvo su expresión más contundente en el incremento e irrupción generalizada de nuevas "villas miserias" y "asentamientos", en la mayoría de las ciudades grandes, medianas y pequeñas- de Argentina.

Cabe acotar que el fenómeno de las "villas" no es nuevo en Argentina y según autores que han estudiado sus orígenes -ver, por ejemplo, Cravino (2006), quien ha realizado una buena sistematización de estos estudios-, se remonta en las grandes ciudades y principalmente en Buenos Aires, a las décadas de 1930 y 1940, como consecuencia de las primeras oleadas de migración campo-ciudad que provocó la incipiente industrialización. Sin embargo, es más propia de las últimas décadas la irrupción masiva de este fenómeno en todos los centros urbanos del país, ocurrida ya no tanto como pro- 
ducto de la industrialización urbana, sino más bien por el impacto de la trasformación estructural de la producción rural, provocada por la profundización de las relaciones capitalistas en esta actividad y que ha generado expulsiones de poblaciones campesinas tradicionales hacia las ciudades del interior del país ${ }^{12}$. A la inversa de antaño, en las últimas décadas no ha sido la industrialización la que atrajo población rural hacia las ciudades, sino que la trasformación estructural de la producción rural expulsó hacia las ciudades a las poblaciones rurales pobres. ${ }^{13}$

Generalmente se denominan "villas" y "asentamientos" a aquellas áreas fuertemente deficitarias, existentes dentro y en las periferias de las ciudades, que por diferentes circunstancias fueron alguna vez ocupadas bajo formas jurídicas ilegales por hogares de bajos recursos, con la finalidad de satisfacer sus necesidades habitacionales básicas, mediante la construcción o auto-construcción de viviendas precarias, que, con el transcurrir del tiempo, por efectos de su forma de producción y de las situaciones de exclusión de sus habitantes, conformaron barrios con formas de enclaves, por diferencias físicas, sociales o culturales, del resto de la ciudad. Las condiciones de los hogares que habitan estas áreas son una de las expresiones más críticas de la pobreza y la marginación urbanas, juntas, por supuesto, a la de aquellos hogares que también siendo pobres, directamente no disponen de vivienda alguna y viven allegados con otros hogares o, peor aún, habitan directamente en la calle.

Sin embargo, el incremento de estas áreas más bien ha tendido oficialmente a no ser visibilizado como problema y a ser sólo registrado por observaciones empíricas locales de estudios particulares laboriosamente llevados adelante por algunos pocos centros de investigación académicos, en distintas ciudades del país, ya que no existen estudios oficiales específicos completos sobre la temática en la Argentina, a la vez que existen importantes subregistros en las diferentes jurisdicciones administrativas. A pesar de esta falta de información, nadie pone en duda que la magnitud de este problema se ha venido incrementando sostenidamente en las últimas décadas en las distintas ciudades del país.

Cravino (2008), por ejemplo, ha analizado este sub-registro oficial e incremento significativo de la problemática en los últimos 25 años en el área metropolitana del Gran Buenos Aires. Por su parte, en

12 Barreto, 2004.

13 Cabe señalar que ésta no es la única causa del fenómeno y aunque no se haya estudiado, se reconoce aquí también como causa a la inmigración desde otros países, como Bolivia, Paraguay, Uruguay y Perú, de trabajadores precarizados sin derechos sociales. 
el caso del área metropolitana del Gran Resistencia, donde trabaja este autor, esta problemática alcanzó valores alarmantes. Según informaciones existentes en el Instituto Provincial de Vivienda del Chaco (IPDUV), en relación con la cantidad de leyes de expropiaciones iniciadas a partir de estos asentamientos desde el año 1973 hasta el 2007, este organismo tiene registrados 100 asentamientos con trámites de leyes de expropiaciones iniciadas, de los cuales 82 ocurrieron a partir de 1995, de forma progresiva en los años consecutivos, con un total de 6.288.004 $\mathrm{m}^{2}$ de superficie de tierras ocupadas y 4139 hogares ubicados en distintos barrios periféricos de dichos municipios. Pero, si se tiene en cuenta el registro de la Unidad Ejecutora del Municipio de Resistencia, que incluye la situación de dominio irregular en tierras municipales y privadas con y sin procesos de expropiación, en sólo este municipio, existían en esa fecha 79 asentamientos en tierras privadas y 82 en tierras municipales. Se estima sólo para los 79 asentamientos en tierras privadas un total de alrededor de 40.000 habitantes, es decir, aproximadamente un $15 \%$ de la población que actualmente tendría este municipio. De acuerdo con diferentes fuentes locales, actualmente se estima que entre un $20 \%$ y un $30 \%$ de la población vive en asentamientos y villas en el AMGR, en condiciones de extrema precariedad. Por otra parte, Mike Davis, en una reciente obra dedicada al tema, tomando como base el estudio global publicado por Naciones Unidas en el año 2003, con el nombre de "The Challenge of Slums. Global Report on Human Settlements 2003" y otros estudios preocupados por dimensionar el fenómeno a escala global, señala que Argentina presenta un 33,1 \% de su población total viviendo en áreas urbanas deficitarias ${ }^{14}$.

La distinción entre "villas" y "asentamientos" fue formulada por autores como Benítez (2000) o Cravino (2006) para diferenciar dos tipos de estas áreas. "Villa" hace referencia a aquellas ocupaciones más antiguas, producidas de manera menos planificada y con menor organización social inicial, mediante asentamientos sucesivos de hogares individuales en situaciones de pobreza crítica, con formas de producción más espontáneas de demarcación de los terrenos, de los accesos a los mismos y de la construcción de las viviendas, que por antigüedad están hoy más densificadas y presentan intrincados sistemas internos de circulación y de diferenciación de los espacios privados y comunes, fuera de las normativas urbanísticas vigentes.

La denominación de "asentamiento", en cambio, se refiere a aquellas ocupaciones más recientemente ocurridas, como producto de acciones colectivas, realizadas por grupos de hogares en situaciones de pobreza crítica, en su mayoría jóvenes, a partir de algún tipo de organización social previa, y que se

14 Davis, 2007: 42 
han asentado con construcciones muy precarias de rápida ejecución, pero con una mayor planificación, teniendo en cuenta muchas veces la situación de dominio de la parcela (para contrarrestar desalojos) y haciendo una subdivisión de terrenos más similar al resto de la ciudad, tratando de seguir en lo posible las exigencias de las normativas urbanas, con vistas a su futura regularización.

Aquí, desde una perspectiva de derechos humanos y con fines de definir intervenciones integrales desde la política, se considera más conveniente hablar de áreas urbanas deficitarias críticas, que de "villas" y "asentamientos", como unidad de estudio para posibles intervenciones, porque si bien es cierto que las "villas" y los "asentamientos" son configuraciones socio-espaciales generalmente bien definidas, por los límites de los terrenos y porque en ellos convergen los peores indicadores de exclusión social, se ha podido observar que en muchos casos -como los del Gran Resistencia- no existen límites contundentes entre estos enclaves marginados y las condiciones de vida de los hogares que viven en el entorno circundante de estas áreas, en la medida en que ellos también en muchos casos

168 revista invi № 69 / Agosto 2010 / Volumen № 25: 161-187 son afectados por procesos de desinversión pública y privada, de desvalorización y de exclusión económica, social y cultural, que dan como resultado la conformación de áreas urbanas más amplias, que presentan grados variables de precariedad del hábitat y de condiciones de vida, que requieren ser observadas con detenimiento a la hora de definir una intervención integral y no "focalizada".

\section{Enfoques predominantes de las respuestas al problema desde la política en las últimas décadas ${ }^{15}$}

Los enfoques de las respuestas a este problema implementadas por el Estado durante el periodo analizado se pueden diferenciar de manera sintética en tres etapas bien diferentes: ${ }^{16}$

a) Hasta los años 80 existieron, al menos, dos visiones contrastantes respecto de la consideración de estos sectores sociales: la de los gobiernos militares por un lado y la de los democráti-

15 En relación al tema de Política Habitacional se han analizado los siguientes trabajos de autores especializados: CUENYA (2006), CLICHEVSKY (2003), FERNÁNDEZ WAGNER (2003 y 2004), RODULFO (2006 y 2009), LENTINI y PALERO (2001 y 2006) y el INFORME MISIÓN DE INVESTIGACIÓN (2004).

16 Esta periodización tiene como sustento conceptual el análisis realizado en BARRETO (2008b). 
cos por el otro -sobre todo la del peronismo-, que pueden sintetizarse en el rechazo o la discriminación en el primer caso, contra la solidaridad o consideración populista en el segundo. No obstante, puede afirmarse que por sobre estas diferencias ha predominado un enfoque centralmente "desarrollista" sobre el problema, tendiente a creer que el crecimiento económico sostenido y la producción industrial masiva y subsidiada de viviendas sociales, erradicarían definitivamente el problema. ${ }^{17}$

b) Durante los años 90, con la profundización de la crisis del Estado keynesiano, en el marco de la Reforma del Estado, del ajuste estructural y de las políticas sociales de "contención" de la crisis por el incremento estructural de la pobreza y la desocupación, este enfoque cambió drásticamente y comenzaron a aplicarse políticas específicas de aceptación e integración de estas áreas a las ciudades, que consideraron tanto la construcción de viviendas nuevas para relocalizar situaciones ambientalmente críticas (áreas inundables, altamente contaminadas, etc.), como acciones de consolidación y mejoramiento habitacional y urbano de áreas

17 En este periodo, Argentina alcanzó a desarrollar un parcial Estado Keynesiano del Bienestar, en el que la producción subsidiada de vivienda masiva formaba parte de otras instituciones sociales públicas (educación, salud, etc.), tendientes a mejorar la calidad de vida de los asalariados formales. Esta política alcanzó su máxima expresión con la creación del FONAVI a mediados de los años 70 . deficitarias críticas existentes, factibles de ser urbanizadas, que incluyeron acciones sociales destinadas a contrarrestar la exclusión. Estas políticas combinaron los aportes alternativos de promoción social implementados en los años 70 por diferentes centros académicos y de cooperación en distintos países del Tercer Mundo, con la noción del "desarrollo social", impulsada por los organismos financieros internacionales que promovieron la Reforma del Estado y el Ajuste Estructural, consistente en la capacitación de los sectores sociales excluidos, para su reinserción en la nueva economía de mercado promovida por este paradigma. Esta política contó mayoritariamente con el financiamiento de los organismos financieros internacionales que impulsaron la Reforma del Estado en Argentina ${ }^{18}$.

c) A partir del colapso del paradigma del "Consenso de Washington", manifestado en la profunda crisis económica, política y social de los años 2001-2002, cambió nuevamente la visión sobre el problema. El gobierno constitucional que asumió en el 2003 rompió con la dependencia de las políticas aperturistas de los organismos internacionales de crédito, impuestas

18 Barreto y Romagnoli, 2007. 
como condición de los financiamientos, e impulsó una reconstrucción del Estado como motor de la economía, promoviendo el desarrollo productivo orientado a los mercados externos e internos, como fuente de generación de empleos y de ingresos más genuinos para implementar políticas sociales subsidiadas hacia los sectores de menores recursos. En este marco, se implementó una política habitacional (y de infraestructuras territoriales) más ambiciosa en lo cuantitativa, cuyo eje central fue la reactivación de la industria de la construcción como generadora de fuentes de empleos y de demandas para el mercado de la construcción. Una buena parte de ella tuvo el carácter de subsidio para los sectores más excluidos, e incluyó tanto la construcción de viviendas nuevas como acciones de mejoramiento habitacional y de urbanización de áreas deficitarias críticas. Esta nueva política volvió a separar las acciones físicas (las obras), de las acciones sociales, tendientes en este periodo básicamente a la promoción del trabajo formal y la producción como sustento de la integración social, diluyéndose la integración de ambas acciones en intervenciones focalizadas de estas áreas, propias del periodo anterior. ${ }^{19}$

19 Cabe acotar que los programas de mejoramiento barrial financiados por el BID (PROMEBA y Rosario Hábitat), iniciados en el periodo anterior, continuaron "solapadamente" durante este periodo.

170 revista invi № 69 / Agosto 2010 / Volumen № 25: 161-187
Sin ánimo de realizar aquí una evaluación exhaustiva de los programas y proyectos de estas políticas,$^{20}$ se pretenden resaltar las siguientes cuestiones:

a) La política promovida durante los años 90 contó con una labor social focalizada, que incluyó algunas formas de participación de los destinatarios, que se considera de cierta validez, para abordar la especificidad del problema desde una política particular para estas áreas y estos sectores sociales excluidos. Sin embargo, se piensa que resultó inadecuada y muy pobre en la calidad de los proyectos y las soluciones físicas y sociales como consecuencia del marco teórico general que guió a las mismas, derivado del modelo macro-estructural en la que ella se inscribió, que trató de focalizar en extremo la inversión social y de lograr la integración social por medio del mercado, en un contexto de profunda crisis de desigualdad social, pobreza y desempleo, y no a partir de una política redistributiva de reconocimiento de derechos, tendiente a una sociedad más equitativa. ${ }^{21}$

b) Por su parte, la política iniciada en el año 2003 impulsó un modelo de desarrollo macro-es-

20 Actividad que es realizada en la labor investigativa desde hace varios años.

21 Un desarrollo más extenso de este análisis puede encontrarse en el análisis del PROMEBA realizado por Barreto y Romagnoli (Ob. Cit.). 
tructural distinto, que buscó reconstruir las instituciones de protección social del Estado como vía de integración social, a partir de impulsar la producción, el empleo formal y el reconocimiento de los derechos sociales básicos de los sectores excluidos, que se lo considera válido, y que le dio a una parte de la política habitacional un sentido interesante, en cuanto que buscó la promoción de los sectores excluidos, mediante su participación en la industria de la construcción y en organizaciones productivas cooperativas. Sin embargo, en este caso, los proyectos y las soluciones, en muchas oportunidades, tampoco han resultado adecuadas para favorecer la integración, debido principalmente a la falta de un modelo conceptual específico de intervención, que supere la visión sectorial de entender al problema exclusivamente como habitacional y a su solución mediante la producción masiva de viviendas individuales en conjuntos nuevos -muchas veces ubicados fuera de los tejidos urbanos consolidados- en desmedro de una decidida y masiva intervención de estas áreas deficitarias críticas, con un fin de integración social.

Con este último fin, a continuación se reflexiona sobre una determinada caracterización del proble-

OPINIÓN: El concepto de "hábitat digno" como meta de una política integral de áreas urbanas deficitarias críticas, para la integración social desde los derechos humanos /

Miguel Ángel Barreto ma y de su abordaje, para fundamentar el concepto de Hábitat Digno como meta de intervención de una política integral de estas áreas para lograr la integración social.

\section{La especificidad del problema y su abordaje desde una política que promueva la integración social a partir del reconocimiento de los derechos humanos}

La especificidad del problema de las áreas urbanas deficitarias críticas desde este enfoque obedece a dos cuestiones: (1) a las condiciones de vida altamente deficitarias en las que viven estos hogares y (2) a la complejidad de factores relacionados que conforman estas condiciones, los cuales demandan un tipo muy especial de intervención, porque no conforman un mero problema habitacional, sino de exclusión multidimensional, en el que la vivienda es sólo un factor más entre otros tantos, íntimamente relacionados entre sí..$^{22} \mathrm{~A}$ efectos de comprender estos factores, se los puede ordenar sintéticamente en las siguientes dimensiones (Tabla 2).

22 Para profundizar sobre este enfoque de la complejidad del problema y las variables que intervienen en él, puede consultarse BARRETO (2006) y BARRETO (2008c). 


\section{SÍNTESIS DE FACTORES DEFICITARIOS PREDOMINANTES EN LAS CONDICIONES DE VIDA EN LAS ÁREAS URBANAS DEFICITARIAS CRÍTICAS SEGÚN LAS DISTINTAS DIMENSIONES}

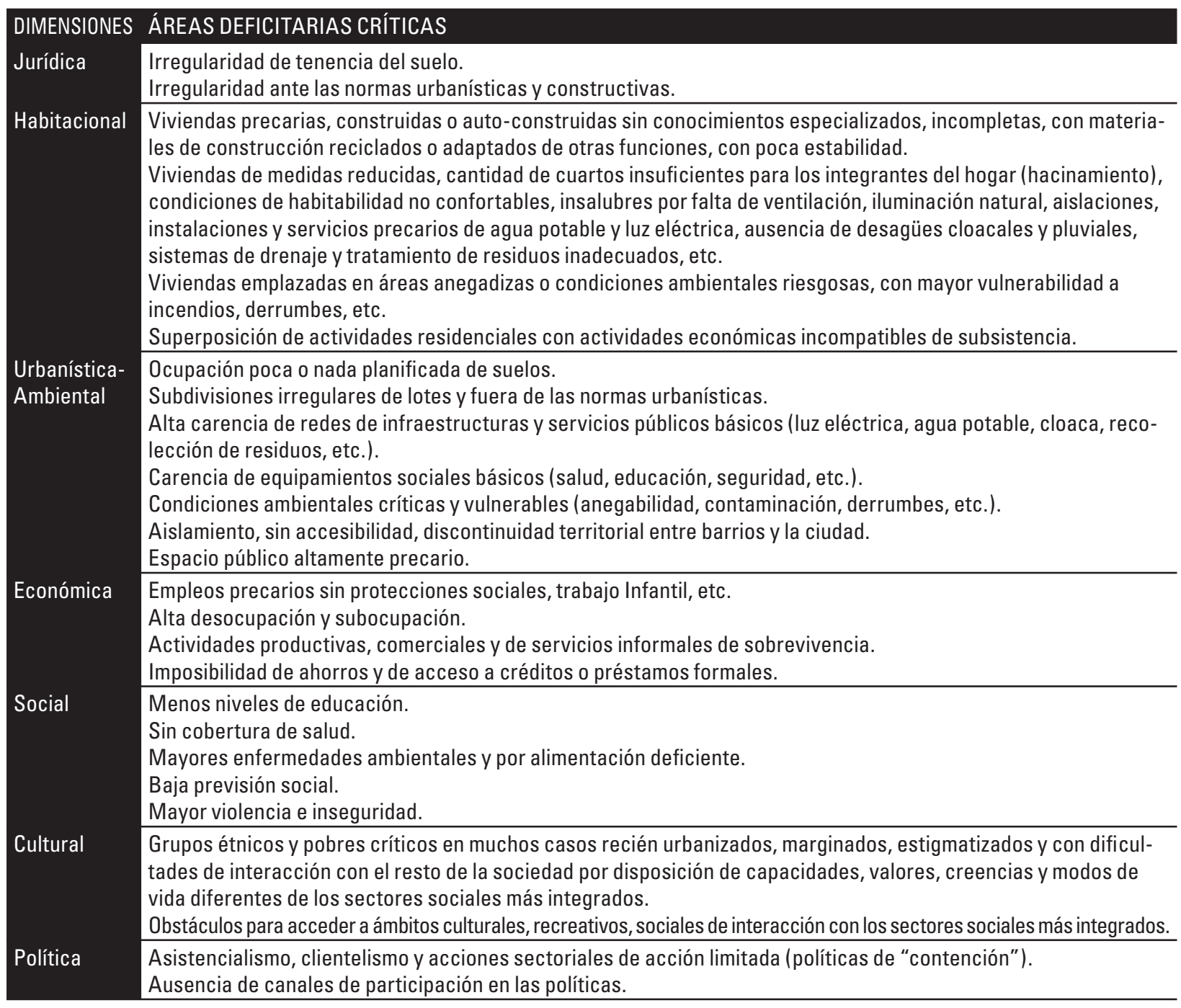

TABLA 2

Elaboración en base a: BARRETO, Miguel; PELLI, Víctor; ALCALÁ, Laura; GIRÓ, Marta; BENíTEZ, Andrea; FERNÁNDEZ, María; PELLI, María y ROMAGNOLI, Venettia. (2009). 
La particularidad y complejidad de este problema consiste en que las combinaciones posibles de estos factores, que se pueden dar en cada uno de los hogares y áreas, se encuentran siempre relacionados entre sí y conforman un círculo de reproducción de las condiciones de vida, que torna muy específica su reversión, porque al estar relacionados entre ellos de diferentes maneras, requieren siempre ser abordados lo más integralmente posible, dado que se indicen mutuamente. Y por el contrario, cuanto más sectorial sea el abordaje de los mismos, más probabilidad existe que la intervención resulte ineficaz para modificar el problema en su conjunto y, por ende, contribuya a la reproducción de estas condiciones.

Por ejemplo, no se le pueden solucionar a estos hogares solamente algunos aspectos de la dimensión habitacional, mediante importantes inversiones en la regularización de dominio de los terrenos o construyendo una vivienda más adecuada, sin tener en cuenta el impacto que estas soluciones producen sobre los otros factores deficitarios que afectan sus condiciones de vida, y tampoco sin tratar de incidir en la reversión de los mismos, porque existe una gran probabilidad de que -al no recibir ningún estímulo para revertir factores tales como el de sus ingresos económicos para hacer frente a los nuevos gastos de la vivienda, o para acceder a

OPINIÓN: El concepto de "hábitat digno" como meta de una política integral de áreas urbanas deficitarias críticas, para la integración social desde los derechos humanos /

Miguel Ángel Barreto alimentos, a atención a la salud o de contrarrestar actitudes de marginación o de accesibilidad a equipamientos sociales de educación, salud, cultura, recreación, etc.- utilicen las mejoras habitacionales como un recurso para paliar problemas más urgentes y terminen desprendiéndose de estas mejoras para aliviar transitoriamente algunos de estos problemas, volviendo a vivir en condiciones iguales a la anterior, reproduciendo sus situaciones de pobreza y tornando ineficiente la acción del Estado. $^{23}$

Por esta razón, es imprescindible que el abordaje al problema relacione a todos los factores que afectan a las condiciones de vida de los hogares de un área, teniendo en cuenta cómo se inciden y retroalimentan mutuamente en cada caso. Este es el principio básico que tiene que guiar una política integral desde una perspectiva de los derechos humanos. De aquí que toda intervención requiere un profundo conocimiento de cada área en todas las dimensiones aludidas, antes de actuar, y de un proyecto integral de intervención que atienda a todas ellas.

Las soluciones en una intervención de estas características, obviamente, deberán ser multidimensionales (inter-disciplinarias), inter-sectoriales e inter-jurisdiccionales, e implicarán acciones de

23 Situaciones como éstas son frecuentemente observadas sobre el terreno en los estudios empíricos realizados en el Área Metropolitana del Gran Resistencia. 
plazos temporales muy diferentes unos de otros en las distintas dimensiones, y también inversiones económicas y de recursos muy divergentes en magnitud, y si bien serán productos medibles y cuantificables, tienen que ser concebidas como desencadenantes de procesos relacionados -en algunos casos a largo plazo- desde una perspectiva de gradualidad y progresividad de resultados. ${ }^{24}$

Además, una intervención así debe tener en cuenta que siempre va a actuar sobre un hábitat que se encuentra socialmente producido, es decir, que va a actuar sobre un proceso en marcha, en el que los hogares generalmente disponen de alguna organización social previa y que, a pesar de las limitaciones de recursos que poseen, sus integrantes disponen de capacidades en el desarrollo de estrategias de supervivencia, que despliegan cotidianamente para vivir y para construir su hábitat. Ellas, encuadradas en un marco de reconocimiento y promoción de derechos humanos, representan un germen importante a capitalizarse como recurso para iniciar un proceso de reversión de estas condiciones.

La formulación de las soluciones tiene que efectuarse a partir de estos procesos en marcha y teniendo en cuenta el trabajo realizado cotidianamente por los hogares y sus organizaciones, para canalizar este potencial en la resolución de los problemas.

24 En cuanto a la progresividad y gradualidad en el caso de las soluciones habitacionales, véase PELLI (2007).
Para que esto suceda, la intervención tiene que ser necesariamente participativa ${ }^{25}$. La participación social plena, encauzada desde una perspectiva de promoción para la construcción de ciudadanía, es una rica vía de formación para que estos hogares puedan superar progresivamente las situaciones de marginación cultural y social ligadas a la precariedad del hábitat en la que se encuentran. En este sentido, debe tenerse en cuenta que en muchos casos, las organizaciones sociales de base existentes en estas áreas son muy activas en la producción cotidiana del hábitat, por lo tanto, es fundamental acordar y articular las acciones con dichas organizaciones, haciendo extensiva la participación en la co-gestión de las soluciones.

\section{El concepto de Hábitat Digno como meta para una política que promueva la integración social a partir de los derechos humanos}

De la misma manera que para solucionar las necesidades exclusivamente habitacionales el Estado reconoce estándares que debe reunir una vivienda digna como meta para intervenir desde las políticas habitacional sectoriales, orientadas en general

25 Pelli, op. cit. 


\section{ESPECIFICIDAD DEL PROBLEMA DE LAS ÁREAS URBANAS DEFICITARIAS CRÍTICAS (AUDC) Y DE SU ABORDAJE INTEGRAL}

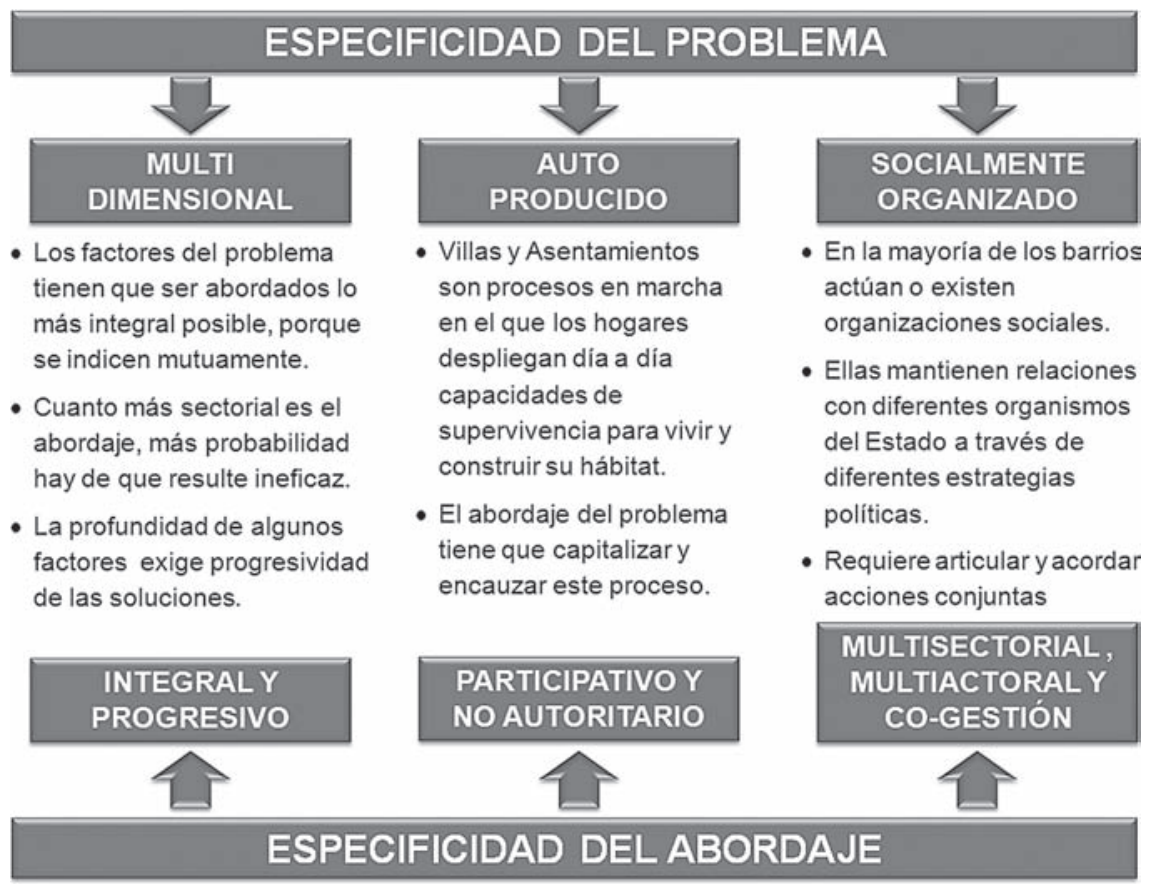

ESQUEMA 1

Fuente: Elaboración BARRETO, Miguel.

a construir viviendas nuevas, la complejidad e integralidad que presenta el problema de los hogares que viven en las áreas urbanas deficitarias críticas -visto desde una perspectiva de reconocimiento de los derechos humanos- obliga a reconocer un mínimo deseable en el conjunto de factores que hacen a sus condiciones de vida como meta de una intervención integral de estas áreas. Para este mínimo deseable se propone aquí el concepto de Hábitat Digno. 
Este concepto obviamente incluye a la vivienda digna, pero la engloba junto a otros factores que en conjunto hacen un mínimo deseable nivel de calidad de vida de los hogares, en conformidad con la integralidad que demanda la interdependencia de los derechos humanos. El mismo incluye a las condiciones habitacionales, pero incluye también condiciones para el ámbito barrial, su relación con la ciudad y los diversos factores que hacen a la integración de estos hogares a la sociedad: la tenencia segura de la vivienda, el acceso al trabajo decente y los recursos económicos necesarios para la reproducción adecuada de la vida, las protecciones sociales para acceder a la salud, la educación, la participación en la vida social y política, la identidad social positiva y las posibilidades de disfrutar del ocio y la cultura, etc., que es necesario que queden lo más especificadamente posible como metas para el diseño de las soluciones de los proyectos integrales a elaborarse para la intervención de dichas áreas.

En lo habitacional, en Argentina la política sectorial tradicional, dedicada a la construcción masiva de viviendas estandarizadas nuevas para los sectores de menores recursos (pero que poseen ciertos vínculos de integración social), tiende a respetar ciertos estándares de calidad para la construcción de las mismas, cumpliendo con lo que el INDEC

176 revista invi № 69 / Agosto 2010 / Volumen № 25: 161-187 define como viviendas adecuadas (Viviendas Tipo A y Departamentos ${ }^{26}$. Sin embargo, a la hora de intervenir en las áreas urbanas deficitarias críticas, a través de programas de mejoramientos barriales y habitacionales, en general brinda soluciones de menor calidad, que perpetúan la desigualdad social (como si a los que se encuentran en situación de pobreza les correspondiera vivir en una vivienda de menor calidad). Ahora bien, al margen de que aquí se piensa que estos estándares merecen ser revisados profundamente y mejorados cualitativamente, se considera que para alcanzar un hábitat digno para los hogares que habitan las áreas urbanas deficitarias, las soluciones habitacionales deben resolverse (aunque fuera por formas diferentes de producción y progresivamente) teniendo como referencia el cumplimiento de estándares mínimos similares a los del resto de la sociedad.

Aunque aquí no se pretende definir especificaciones técnicas con relación a estos estándares mínimos, se considera que para mejorar estas áreas, las viviendas -individuales y colectivas- tienen que ser intervenidas para cumplir (progresivamente) con los requisitos de quedar construidas según las reglas del arte, ser estables, duraderas, aislantes de las agresiones e inclemencias climáticas, contar con ventilación e iluminación natural adecuada

26 Entre otras normas, la Dirección de Políticas Habitacionales del gobierno nacional (SDUyV-MPFIPyP), en la actualidad exige el cumplimiento de "Estándares mínimos de calidad para viviendas de interés social". 
de sus ambientes, contar con las instalaciones para todos los servicios básicos, prever dormitorios suficientes para que los integrantes del hogar dispongan de intimidad, contar con lugares para cocinar los alimentos y la higiene personal. Pero además de todo esto, deben ser cómodas y ajustadas a las necesidades básicas particulares de los hogares, a sus formas de vida, dar cabida para expresar sus identidades, y aunque inicialmente sean mínimas, estar pensadas para crecer o evolucionar según las trayectorias particulares de los hogares, ser seguras al vandalismo, disponer de un lugar para reuniones familiares y amistades, contar con espacios de trabajo en casos necesarios, prever lugares para guardar medios de transportes, etc. En resumen, estar resueltas a partir de estándares mínimos similares, pero con flexibilidad a las particularidades de los hogares. En Argentina esta intervención todavía es posible de realizar en la mayoría de los casos, dado que la incidencia histórica del Estado en el desarrollo urbano ha evitado que las áreas urbanas deficitarias críticas adquieran los tamaños y las densidades que las mismas presentan en países como Brasil, México, Perú o Venezuela. Con excepción de algunas villas de Buenos Aires, como "Villa 31" o "La Cava", la mayoría de las existentes en el resto del país en general son de una sola planta de altura y con densidades aún factibles de intervenir en el mejoramiento habitacional y urba-

OPINIÓN: El concepto de "hábitat digno" como meta de una política integral de áreas urbanas deficitarias críticas, para la integración social desde los derechos humanos /

Miguel Ángel Barreto no. Quedan exceptuados, por supuesto, los casos de traslados por problemas ambientales (anegabilidad, contaminación, derrumbe, etc.), legales, etc.

A escalas barrial e inter-barrial, las soluciones de las políticas habitacionales de Argentina también han sido deficientes ${ }^{27}$, tanto en las intervenciones orientadas a construir conjuntos habitacionales nuevos, como en las dedicadas a mejoramientos barriales y habitacionales. Con frecuencia, la dimensión urbana directamente es omitida en las mismas, descuidando lo que sus soluciones representan en el mejoramiento de la calidad de vida de estos hogares y lo que una inserción territorial adecuada contribuye en la integración social de los mismos. Para alcanzar un hábitat digno desde la perspectiva que aquí se promueve, estas escalas deben resolverse realizando un profundo reconocimiento de lo que ellas representan en ambos sentidos. Sin entrar aquí a citar los extensos aportes realizados por la sociología y la antropología sobre los barrios ni tampoco a analizar las polémicas entabladas al respecto sobre el concepto de comunidad en las sociedades capitalistas, no hay que dejar de reconocer, en torno al espacio doméstico de las viviendas, la existencia de un área de transición con la ciudad, que es apropiada por las personas en la cotidianeidad y que tiene tanto un profundo sentido de sociabilidad como de complementarie-

27 Barreto, Benítez, Fernández, Giró y Zavala (2007) Barreto, Alcalá, 2008. 
dad en la satisfacción de numerosas necesidades básicas. Más aún en las áreas urbanas deficitarias críticas, en las que la vida barrial es muchas veces tanto la "extensión" de la vida doméstica como el sustento de importantes redes sociales de intercambios, que contribuyen a la sobrevivencia.

Una política integral para estas áreas, desde la perspectiva aquí propuesta, debe brindar calidad de vida y dar plenas posibilidades a las relaciones de la vida social-comunitaria. Para esto, los barrios deben contar con calles y veredas que se puedan transitar con comodidad peatonalmente y con los diferentes medios de transportes -aun cuando llueve-y tener iluminación para hacerlo de noche; disponer de plazas o espacios públicos con espacios para reuniones sociales y culturales, charlar en grupo, descansar o practicar deportes, de acuerdo con las necesidades de los diferentes grupos etarios y de género (niños, adolescentes, adultos y mayores, de ambos géneros), disponer de equipamientos, iluminación y vegetación. Es fundamental que el barrio cuente con servicios de recolección de residuos y transporte público, que en él o en su entorno próximo haya jardines de infantes, guarderías, escuelas primarias, lugares de atención a la salud y de seguridad, que no existan focos de contaminación de ningún tipo, ni riesgos ambientales que afecten la salud de las personas, etc., que se encuentren en

178 revista invi № 69 / Agosto 2010 / Volumen № 25: 161-187 relación de integración con otros barrios, el centro y los equipamientos de la ciudad, etc. ${ }^{28}$. Además, el diseño de todos estos elementos es relevante, tanto en lo atinente a la identidad como a la integración social, ya que las intervenciones de las políticas habitacionales -tal como se señaló- tienden generalmente a homogeneizar los diferentes colectivos con respuestas similares, a la vez que a estigmatizarlos con elementos de menor calidad respecto de los de los sectores sociales más integrados.

En cuanto a las dimensiones social y cultural, se han ensayado hasta hoy distintas experiencias en las políticas de mejoramientos barriales y de viviendas, orientadas a los sectores excluidos en Argentina. Como se ha analizado someramente en el tercer punto, las que surgieron en los años 90 tuvieron un encuadre macro-estructural inadecuado que fue ineficaz y muy limitado para la integración social, a pesar de la buena intención de promoción y participación social focalizada que realizaron. En cambio, las políticas actuales, casi inversamente, se insertan dentro de una mejor orientación macro-estructural desde la perspectiva que aquí se promueve, pero -y aunque sea muy pronto para evaluarlas con seriedad en sus resultados focalizados- se considera que no han acertado aún debidamente en esta escala.

Para alcanzar un hábitat digno es imprescindible -como ya se señaló en Barreto y Benítez (2008)-

28 Barreto y Alcalá, 2008. 
que los hogares que habitan estas áreas (como el resto de los excluidos) avancen hacia una progresiva inclusión en un sistema de protección social lo más universal posible, para que se nivelen sus condiciones mínimas de calidad de vida con las de los sectores más integrados de la sociedad. Al respecto hay que tener en cuenta que en Argentina, la falta de protección social tiene como causa central que los derechos sociales propios de la ciudadanía no se encuentran directamente relacionados con la posesión de los derechos civiles que definen su pertenencia a la sociedad, sino sólo a los empleos registrados y formalizados; por lo tanto, aspectos muy básicos, que hacen a la calidad de vida, tales como el acceso a la atención sanitaria, el acceso a la jubilación, la regulación de la jornada de trabajo, dependen indefectiblemente de acceder a un empleo de este tipo, por no mencionar otros derechos, tales como el de la vivienda adecuada y a la ciudad, etc., ya que el acceso a los servicios urbanos básicos se halla ligado a esta cuestión, y son esenciales para la integración social. Todo esto afecta tanto a las condiciones de vida de estos hogares como a sus posibilidades de relacionamiento igualitario con el conjunto del tejido social.

Sin embargo, por más que a nivel estructural se haga un esfuerzo para facilitar el acceso a empleos más formales, que brindan protección social, y se les reconozcan algunas protecciones básicas universales que no dependan de la situación laboral -como está ocurriendo en algunos aspectos en Ar- gentina (jubilación, protección a la niñez, etc.)-, ello aún no es suficiente. Hay que tener en cuenta que la mayoría de los hogares que habitan estas áreas han vivido siempre o por varias generaciones en el desamparo de una protección social igualitaria y del acceso a servicios básicos adecuados (como la educación) y tanto este hecho, que los ha obligado a vivir en la subsistencia o la dependencia asistencialista, como el origen de donde provinieron en muchos casos, no les permitieron aún desarrollar capacidades necesarias para lograr una inserción adecuada en el medio social urbano más integrado, disponiendo de estructuras mentales internas muy limitantes en este sentido. Por esta razón, para alcanzar a conformar un hábitat digno, estos hogares necesitan contar con una promoción externa particularizada, que contribuya a disminuir estas diferencias de capacidades con los sectores más integrados de la sociedad. También debe tenerse en cuenta que la exclusión afecta con mayor fuerza a los segmentos más débiles de estos hogares, como menores, mayores, mujeres, personas con capacidades diferentes, grupos étnicos originarios y sectores pobres recientemente urbanizados, que disponen aún de menor capacidad de desempeño en el medio urbano, y cuyas situaciones de exclusión se ven agravadas por tratos discriminatorios o estigmatizadores de los sectores dominantes, que afectan aún más sus posibilidades de acceder a una inserción en el sector más formal de la sociedad y en particular a empleos formales 
de mejor calidad. Por lo tanto, para alcanzar un hábitat digno, estos hogares requieren tener a su alcance un apoyo externo particularizado y sostenido en el tiempo, que les ayude a contrarrestar a nivel local comunitario los factores que afectan sus condiciones de vidas y su exclusión de la sociedad.

En resumen, estos hogares deben poder contar con políticas "macro-sociales" y "micro-sociales"29, siendo las primeras las relacionadas con la restitución de los derechos de la ciudadanía, propias del Estado de Bienestar, que fueron afectadas fuertemente en las últimas décadas, y las segundas las políticas de intervención directa que trabajan desde "adentro" los emergentes de la exclusión, a partir de perspectivas que sean de comprensión de cómo los afectados perciben sus problemas y las potencialidades y posibilidades que tienen para solucionarlos, teniendo en cuenta los ejes de la educación, la salud, la seguridad, la cultura y, por supuesto, el trabajo decente. Estos hogares tienen que disponer de una promoción especial y permanente para suplir las deficiencias que los separa de los sectores integrados de la sociedad, en relación con la menor formación educativa; las deficiencias nutricionales y sanitarias; la exposición a la violencia e inseguridad; a las patologías derivadas de la exclusión social, a las estigmatizaciones y la carencia de canales de participación política, etc. Esto involucra obviamente la superación de las políticas sociales instaladas en las últimas décadas, de tipo asistencialistas y clientelares, tendientes a reproducir las desigualdades y las situaciones de exclusión.

Es necesaria una principal consideración a las actividades económicas, porque más allá de las políticas generales de promoción de empleos y trabajos formales, los hogares que habitan estas áreas deben poder contar con apoyo especial para revertir la informalidad de las numerosas actividades productivas, comerciales y de servicios que practican para subsistir. Esto sólo puede lograrse mediante capacitación, apoyo financiero, formalización y tutorías permanentes para la inserción en los mercados, la generación de demandas, el sostenimiento de precios, la reconversión de actividades denigrantes o no deseables, etc. En este campo es posible también capitalizar las capacidades relacionadas con el mejoramiento del propio hábitat para la generación de fuentes de empleos e ingresos formales, como está intentando en algunos casos la actual política habitacional argentina, mediante producción cooperativa y empresas sociales.

Finalmente, en las dimensiones jurídica y política, la particularidad de las condiciones de vida de estos hogares se caracteriza por la ilegalidad o la precariedad de tenencia del suelo y las viviendas, y el predominio de políticas sociales asistencialistas, clientelares y de contención social. En la pri-

29 Carballeda, 2005. 
mera de estas dimensiones, porque la propiedad de las tierras pertenecen a agentes privados o al propio Estado, ya sea porque son tierras que pertenecieron a algún organismo público que entró en desuso (FF.CC., Ejército, etc.) o porque -como es habitual en Argentina- el Estado las expropió para solucionar el problema del propietario privado, sin concretar nunca la tenencia segura a favor de los ocupantes. Este no es espacio para proponer o discutir medidas concretas al respecto, ya que se trata de un tema complejo y en diferentes ámbitos se encuentra en discusión la manera de regular la propiedad privada del suelo en función social, para ponerla al servicio de un desarrollo urbano más equitativo. ${ }^{30}$ Pero, sí es importante señalar que la inseguridad en la tenencia de la vivienda es una de las cuestiones centrales que condiciona el desarrollo personal de los hogares y es necesario avanzar en medidas para revertir esta situación para lograr la integración social. En relación a las políticas sociales, ellas deben encauzarse a través de la participación hacia la capacitación para la inserción y la protección social. ${ }^{31}$

30 En Argentina existen en la actualidad varios proyectos de leyes presentados al parlamento para regular esta situación.

31 Si bien esta política corresponde a un abordaje particularizado de las AUDC, la misma debe darse en un marco de políticas macro-estructurales consistentes con todos los derechos humanos. Con respecto a las escalas y niveles de la política, ver: Elaboración en base a: BARRETO, Miguel; PELLI, Víctor; ALCALÁ, Laura; GIRÓ, Marta; BENÍTEZ, Andrea; FERNÁNDEZ, María; PELLI, María y ROMAGNOLI, Venettia. (2009).

\section{Conclusiones}

El propósito principal de este trabajo es contribuir a reformular el abordaje del problema habitacional de los sectores sociales urbanos más desfavorecidos, presente en gran parte de las políticas habitacionales implementadas en Argentina en las últimas décadas.

Argumenta este propósito la ineficacia que dicha política ha demostrado para contrarrestar la permanencia e incremento de las Áreas Urbanas Deficitarias Críticas (AUDC) en las últimas décadas, que tuvieron como telón de fondo un acelerado proceso de urbanización y concentración urbana, y como detonantes las políticas macro-económicas iniciadas en los años 70, que trajeron aparejada desde entonces una permanencia variable pero constante de altos valores de pobreza, desocupación y desigualdad.

Desde la mirada teórica del artículo, se identificaron durante este periodo tres etapas en el enfoque de las respuestas al problema desde las políticas habitacionales y se consideró que todas ellas han resultado ineficaces. Mientras la política de los 


\section{SÍNTESIS DE LAS METAS DE UNA INTERVENCIÓN INTEGRAL DE LAS AUDC EN LAS DISTINTAS DIMENSIONES PARA ALCANZAR UN HÁBITAT DIGNO}

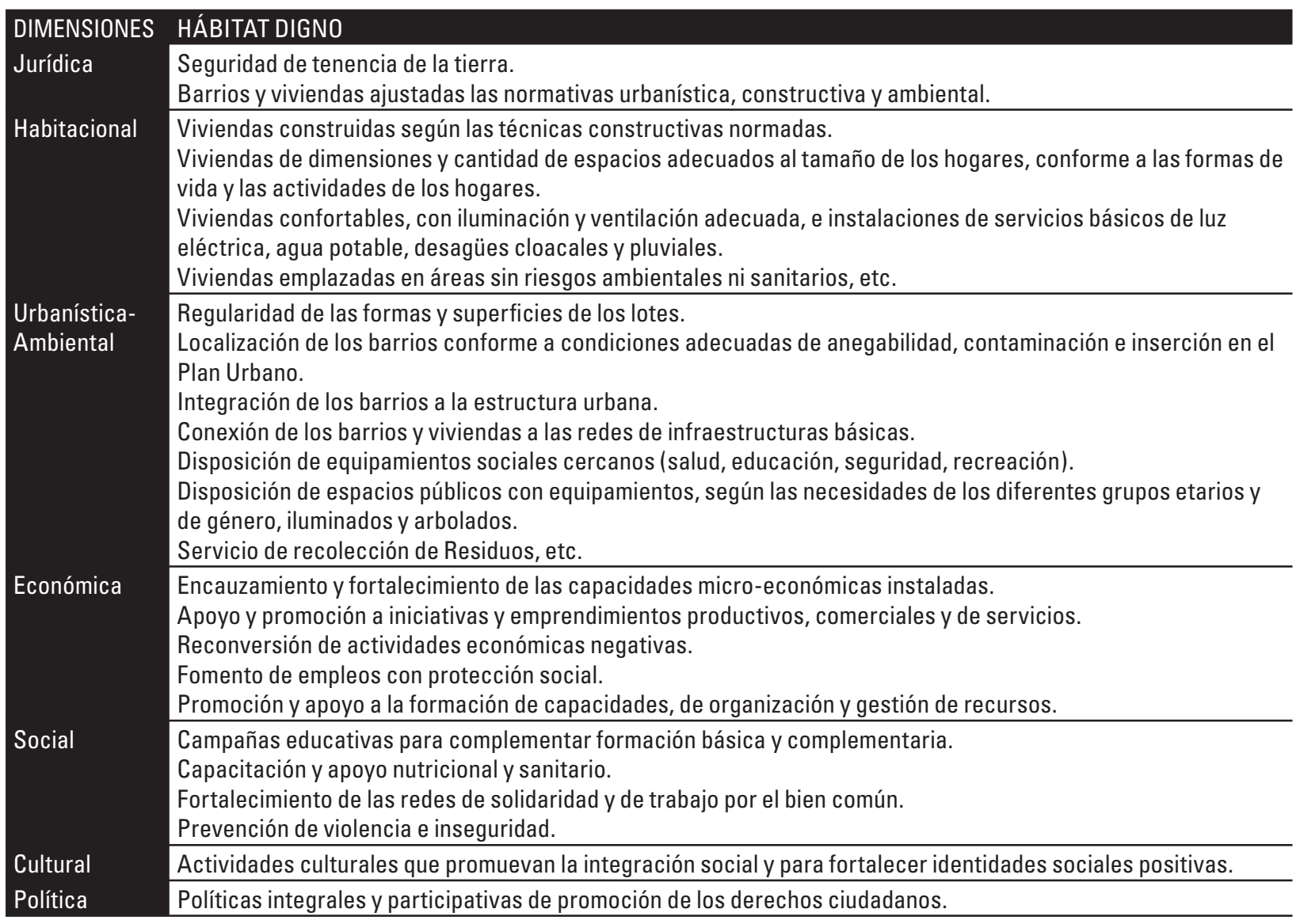

TABLA 3

Fuente: Elaboración en base a: BARRETO, Miguel; PELLI, Víctor; ALCALÁ, Laura; GIRÓ, Marta; BENÍTEZ, Andrea; FERNÁNDEZ, María; PELLI, María y ROMAGNOLI, Venettia. (2009). 
años 90 tuvo algunos aciertos al promover un abordaje específico del problema, fracasó en su excesiva focalización y en su modelo macro-estructural contraproducente para la integración social. En cambio, la política habitacional actual se inscribe dentro de un modelo más favorable en este sentido, pero carece de un enfoque conceptual específico de intervención y evidencia una excesiva visión sectorial del problema, centrada preponderantemente en la producción masiva de viviendas individuales nuevas, sin mejorar integralmente el problema de las Áreas Urbanas Deficitarias Críticas.

El enfoque del problema que promueve este trabajo tiene como base dos nociones: a) la perspectiva integradora postulada por los derechos humanos, conquistados y consagrados en distintas legislaciones y pactos internacionales, y que tal como señala la Carta Mundial por el Derecho a la Ciudad, establece una independencia entre los derechos civiles, políticos, sociales, económicos, culturales, ambientales, etc., y b) considera a la integración social como una situación en la que los miembros de los hogares de una sociedad (cualquiera sea su condición de edad, género o étnica) estén insertos dentro del sistema formal (en condición activa o pasiva) y cuenten con protecciones sociales básicas para acceder a los servicios sociales esenciales, de salud, educación, vivienda, seguridad, ocio y recreación, etc., de manera adecuada en relación con las condiciones de vida generales de la sociedad de pertenencia.
Desde esta perspectiva se considera que el problema de las Áreas Urbanas Deficitarias Críticas tiene una especificidad que obedece a las condiciones de vida altamente deficitarias en las viven estos hogares y a la complejidad de factores relacionados que componen estas condiciones, los cuales demandan un tipo muy especial de intervención, porque no conforman un mero problema habitacional, sino de exclusión multidimensional, en el que la vivienda es sólo un factor más entre tantos otros, íntimamente relacionados, que abarcan las dimensiones: jurídica, habitacional, urbanística-ambiental, económica, social, cultural y política. Desde este enfoque se considera que cuanto más sectorial es el abordaje de estos factores, más probabilidad existe que la intervención resulte ineficaz para modificar el problema en su conjunto y, por ende, contribuya a la reproducción de sus condiciones de vida.

Por esta razón, es imprescindible que el abordaje del problema de los hogares que habitan estas áreas, relacione a todos los factores que afectan a sus condiciones de vida, teniendo en cuenta cómo se inciden y retroalimentan mutuamente en cada caso. Este es el principio básico que debe guiar una política integral desde una perspectiva de los derechos humanos.

Con esta finalidad y de la misma manera que las políticas habitacionales sectoriales reconocen estándares que debe reunir una vivienda digna como meta de sus intervenciones, se propone el concep- 
to de Hábitat Digno, como objetivo de una intervención integral de estas áreas, con la finalidad de englobar en una única noción un conjunto de condiciones mínimas de vida, que deberían ser garantizadas a todos los hogares que las habitan, para avanzar hacia una mayor integración de la sociedad. Esta noción obliga a reconocer un mínimo deseable en el conjunto de los factores intervinientes.

Este concepto incluye a las condiciones habitacionales, pero incluye también condiciones para el ámbito barrial, su relación con la ciudad y los diversos factores que hacen a la integración de estos hogares a la sociedad: la tenencia segura de la vivienda, el acceso al trabajo decente y los recursos económicos necesarios para la reproducción adecuada de la vida, las protecciones sociales para acceder a la salud, la educación, la participación en la vida social y política, la identidad social positiva y las posibilidades de disfrutar del ocio y la cultura, etc., que es necesario que queden lo más especificadamente posible como metas para el diseño de las soluciones de los proyectos integrales a elaborarse para la intervención de dichas áreas.

Excede a los límites de este trabajo desarrollar en detalle instrumentos de esta política integral. ${ }^{32}$ Sin embargo, se han señalado algunas ideas rectoras al respecto, que se remarcan para finalizar:

32 Labor en realización en los actuales proyectos de investigación
- Al ser multidimensionales (e inter-disciplinarias) las soluciones de una intervención de estas características, las políticas tienen que ser necesariamente inter-sectoriales e inter-jurisdiccionales.

- El alcance de sus metas implica acciones de plazos temporales muy diferentes unos de otros e inversiones económicas y de recursos muy divergentes en magnitud, de modo que tienen que ser concebidas como desencadenantes de procesos relacionados -en algunos casos a largo plazo- desde una perspectiva de gradualidad y progresividad de resultados.

- Al tratarse de una intervención de un hábitat socialmente producido, cuyos hogares generalmente disponen de alguna organización social previa y ciertas capacidades en el desarrollo de estrategias de supervivencia, es imprescindible que la política las reconozca como un germen importante a capitalizarse como recurso para iniciar un proceso de reversión de estas condiciones. Las organizaciones sociales de base existentes en estas áreas son muy activas en la producción cotidiana del hábitat, por lo tanto, es fundamental acordar y articular acciones, dándoles participación en la co-gestión de las soluciones.

- Por estas razones las intervenciones tienen que ser necesariamente participativas, encauza- 
das dentro de una perspectiva de promoción y construcción de ciudadanía, como vía de formación para que estos hogares puedan superar progresivamente las situaciones de marginación económica, cultural y social ligadas a la precariedad del hábitat en la que se encuentran.

\section{Bibliografía}

ALTIMIR, OSCAR Y BECCARIA, LUIS. El mercado de trabajo bajo el nuevo régimen económico en Argentina y Distribución del ingreso en la Argentina. En: HEYMANN, Daniel y KOSACOFF, Bernardo (editores). La Argentina de los noventa. Desempeño económico en un contexto de reformas (Tomo I). Buenos Aires, Argentina. EUDEBA-CEPAL. 2000. p. 331-519. ISBN 950-23-1136-1.

BARRET0, MIGUEL. La perspectiva integradora de los derechos. Actuar desde las disciplinas pensando desde la complejidad. En: Encuentro de la Red ULACAV (XIII, 2007, Valparaíso, Chile). Santiago, Chile, Instituto de la Vivienda de la Facultad de Arquitectura y Urbanismo de la Universidad de Chile y Subsecretaría de Desarrollo Regional y Administrativo, Ministerio del Interior, Gobierno de Chile, 2008 (a), pp. 69-76. ISBN 978-956-19-0608-2.

------ Análisis comparativo de supuestos de la política habitacional argentina de la reforma del estado destinada a los afectados por la pobreza. Área Digital, Estudios Territoriales [En línea], 8 (9), febrero 2008 (b). ISNN 1666-4191. [Fecha de consulta: 12 de diciembre de 2009]. Disponible en: <http://arq.unne.edu.ar/publicaciones/areadigital/areall/principal/conjuntomarcosl1.htm>.

------ Un marco epistémico posible para la formulación de una política integral del hábitat para los afectados por situaciones de pobreza crítica en el AMGR [CD]. En: Encuentro de Geohistoria Regional (XXVIII, 2008, Resistencia, Argentina). Instituto de Investigaciones Geohistóricas - CONICET, 2008 (c). ISBN 978-987-21984-5-9.

------ La ínterdisciplina en el abordaje académico del hábitat social "informal", fundamentos, líneas de acción y obstáculos a partir de la carrera de arquitectura. Revista INVI, 21 (56): 16-30. Mayo 2006. ISSN 0718-1299.

------ Bases teóricas y ajuste del objeto de estudio. Documento interno del Proyecto de Investigación PI 041, Secretaría General de Ciencia y Técnica, Universidad Nacional del Nordeste (no publicado). 2005.

------ Transformaciones de la vida urbana de Posadas y Resistencia a fines de los años noventa. Un estudio sobre la dimensión simbólico-ideológica del espacio urbano público. Tesis doctoral. Programa de postgrado en Antropología Social, Universidad Nacional de Misiones, Argentina. 2004.

BARRETO, MIGUEL Y ALCALÁ, LAURA. Cambios en las prestaciones urbano-ambientales de la política habitacional argentina. Reflexiones a partir de cuatro programas orientados a población en situación de pobreza del Gran Resistencia. En: Jornada Internacional de Vivienda Social. (5, 2007, Valparaíso, Chile). Santiago, Chile. Instituto de la 
Vivienda. Facultad de Arquitectura y Urbanismo, Universidad de Chile y Subsecretaría de Desarrollo Regional y Administrativo, Ministerio del Interior, Gobierno de Chile. 2008, pp. 51-63. ISBN 978-956-19-0589-4.

BARRETO, MIGUEL Y BENÍTEZ, ANDREA. Lineamientos en la dimensión sociocultural para una política habitacional integral orientada a situaciones de pobreza crítica del AMGR [CD]. En: Encuentro de Geohistoria Regional (XXVIII, 2008, Resistencia, Argentina). Instituto de Investigaciones Geohistóricas - CONICET, 2008. ISBN 978-987-21984-5-9.

BARRETO, MIGUEL; et al. Aislamiento territorial de la pobreza urbana. Política habitacional social de los '90 en la Argentina: el caso "Ciudad de los Milagros" (AMGR, Chaco). Ciudad Y Territorio, Estudios Territoriales. XXXIX (154): 727-749. Invierno 2007. ISNN 1133-4762.

BARRETO, MIGUEL; et al. Dimensiones, escalas y niveles de las soluciones del problema del hábitat informal desde la perspectiva compleja de una política habitacional integral. En: Seminario Iberoamericano de Ciencia y Tecnología para el Hábitat Popular (III ${ }^{\circ}$ 2008, Córdoba, Argentina) Buenos Aires, Argentina, NOBUKO. 2009, pp. 205-216. ISBN 978-987-584-238-0.

BARRETO, MIGUEL Y ROMAGNOLI, VENETTIA. Programa de Mejoramiento Barrial de Argentina (PROMEBA) ¿Un avance en la gestión oficial del hábitat informal? En: Seminario Iberoamericano de Ciencia y Tecnología para el Hábitat Popular ( ${ }^{\circ}, 2006$, Córdoba, Argentina). Buenos Aires, Ar- gentina, NOBUKO. 2007, pp. 217-238. ISBN 978987-584-113-0

BASUALDO, EDUARDO. Concentración y centralización del capital en la Argentina durante la década del noventa. Buenos Aires, Argentina, Universidad Nacional de Quilmes. 2002. 270 p. ISBN 987-917-347-3.

BENÍTEZ, ANDREA. De la villa al barrio. Hipótesis sobre la construcción de la identidad de los ocupantes de tierra urbana en Resistencia, Chaco [CD]. En: Congreso Argentino de Antropología Social (IV, 2000, Mar del Plata, Argentina). Colegio de Graduados de Antropología y la Facultad de Ciencias Sociales, Universidad Nacional de Mar del Plata. 2000. ISBN: 978-9136-96-9.

CARBALLEDA, ALFREDO. La intervención en lo social. Exclusión e integración en los nuevos escenarios sociales. Buenos Aires, Argentina, PAIDÓS. 2005. 164 p. ISBN 950-12-4514-4.

CARTA MUNDIAL POR EL DERECHO A LA CIUDAD. [En línea]. [Fecha de consulta: 10 de diciembre de 2009]. Disponible en: <http://www.hic-al.org/documentos.cfm>.

CRAVINO, MARÍA CRISTINA. Magnitud y crecimiento de las villas y asentamientos en el Área Metropolitana de Buenos Aires en los últimos 25 años [CD]. En: Encuentro de la Red ULACAV (XIV, 2008, Buenos Aires, Argentina). Facultad de Arquitectura, Universidad Nacional de Buenos Aires. 2008. ISBN 978-950-29-1100-7.

----- Las Villas de la ciudad. Mercado e informalidad urbana. Los Polvorines, Buenos Aires, Argentina, 
Ed. Universidad Nacional de General Sarmiento, 2006. 280 p. ISBN 987-9300-86-6.

CLICHEVSKY, NORA. Pobreza y acceso al suelo urbano. Algunos interrogantes sobre las políticas de regularización en América Latina. CEPAL, Serie Medio Ambiente y Desarrollo (75), p. 84, diciembre 2003. ISSN 1564-4189.

CUENYA, BEATRIZ. Cambios, logros y conflictos en la política de vivienda en Argentina hacia fines del siglo XX. AREA. Agenda de reflexión en arquitectura, diseño y urbanismo (12): 61-71, septiembre 2006. ISSN 0328-1327.

DAVIS, MIKE. Planeta de ciudades miserias. Madrid, España, Ed. FOCA, 2007. 284 p. ISBN 978-8495440-96-9.

FERNÁNDEZ WAGNER, RAÚL. Los programas de mejoramiento barrial en América Latina. [En línea]. URBARED. Presentación del caso. 2003. [Fecha de consulta: 12 de diciembre de 2009]. Disponible en: <http://www.urbared.ungs.edu.ar/download/documentos/Programas\%20de\%20mejoramiento\%20barrial\%20en\%20America\%20Latina. doc>.

------ La construcción y desconstrucción histórica de lo social en el acceso a los bienes y servicios del hábitat. Boletín del Instituto de la Vivienda, 19 (50): 13-22. Mayo 2004. ISSN 0716-5668.

INFORME MISIÓN DE INVESTIGACIÓN. El Derecho a la Vivienda en Argentina. Desafíos para la promoción del derecho a la vivienda y a la tierra en Argentina. Ginebra, Suiza, Centro de derecho a la vivienda y contra los desalojos (COHRE), 2004, p. 114. ISBN 92-95004-33-7.

LENTINI, MERCEDES Y PALERO, DELIA. Descentralización de la política habitacional y gestión territorial. Boletín del Instituto de la Vivienda, 16 (42): 61-71. Mayo 2001. ISSN 0716-5668.

------ Política habitacional comparada: Mendoza y San Luis en el marco de la descentralización. Revista INVI, 21 (56): 117-138. Mayo 2006. ISSN 07181299.

PELLI, VÍCTOR SAÚL. Habitar, participar, pertenecer. Acceder a la vivienda - incluirse en la sociedad. Buenos Aires, Argentina, NOBUKO, 2006, p. 236. ISBN 978-987-584-077-5.

RODULFO, MARÍA BEATRIZ. Las transformaciones urbanas y las políticas de intervención habitacional en Argentina [CD]. En: Encuentro de la Red ULACAV (XII, 2006, Mendoza, Argentina). Mendoza, Argentina. Facultad de Arquitectura, Urbanismo y Diseño, Universidad de Mendoza. 2006.

------ Política habitacional. La lógica de los actores y la dinámica de los programas en la post crisis [CD]. En: Seminario Iberoamericano de Ciencia y Tecnología para el Hábitat Popular (IVº 2009, Córdoba, Argentina). Córdoba, Argentina. Asociación de Vivienda Económica; Centro Experimental de la Vivienda Económica; Universidad Católica de Córdoba e Instituto de Tecnología y Vivienda, Facultad de Arquitectura, Universidad Nacional de Córdoba. 2009. ISBN 978-98722554-5-9.

revista invi № 69 / Agosto 2010 / Volumen № 25: 161-187 187 